\title{
BMJ Open Baggage handler seniority and musculoskeletal symptoms: is heavy lifting in awkward positions associated with the risk of pain?
}

\author{
Stine Hvid Bern, ${ }^{1}$ Charlotte Brauer, ${ }^{1}$ Karina Lauenborg Møller, ${ }^{2}$ Henrik Koblauch, ${ }^{3}$ \\ Lau Caspar Thygesen, ${ }^{2}$ Erik Bruun Simonsen, ${ }^{3}$ Tine Alkjær, ${ }^{3}$ Jens Peter Bonde, ${ }^{1}$ \\ Sigurd Mikkelsen ${ }^{1}$
}

To cite: Bern SH, Brauer C, Møller KL, et al. Baggage handler seniority and musculoskeletal symptoms: is heavy lifting in awkward positions associated with the risk of pain?. BMJ Open 2013;3:e004055. doi:10.1136/bmjopen-2013004055

- Prepublication history for this paper is available online. To view these files please visit the journal online (http://dx.doi.org/10.1136/ bmjopen-2013-004055)

Received 18 September 2013 Revised 28 October 2013 Accepted 1 November 2013

CrossMark

\begin{abstract}
${ }^{1}$ Department of Occupational and Environmental Medicine, Bispebjerg University Hospital, Copenhagen, Denmark

${ }^{2}$ National Institute of Public Health, University of Southern Denmark, Copenhagen, Denmark ${ }^{3}$ Department of Neuroscience and Pharmacology, University of Copenhagen, Copenhagen, Denmark
\end{abstract}

Correspondence to Stine Hvid Bern; stinehvid@gmail.com

\section{ABSTRACT \\ Objectives: Heavy lifting is associated with musculoskeletal disorders but it is unclear whether it is related to acute reversible effects or to chronic effects from cumulated exposure. The aim of this study was to examine whether musculoskeletal symptoms in Danish airport baggage handlers were associated with their seniority as baggage handler, indicating chronic effects from cumulated workload.}

Methods: We established a group of baggage handlers employed at Copenhagen Airport during the period 1983-2012 ( $n=3092)$ and a reference group of men in other unskilled occupations with less heavy work $(\mathrm{n}=2478)$. Data regarding work history, lifestyle and musculoskeletal symptoms were collected using a self-administered questionnaire (response rate $70.1 \%$ among baggage handlers and $68.8 \%$ among the reference group).

Results: The ORs of self-reported musculoskeletal symptoms during the last 12 months in the neck/upper back, lower back, shoulders, elbows, wrists, hips and knees were significantly higher in baggage handlers than in the reference group. These differences were explained by significant linear effects of baggage handler seniority for six anatomical regions. Adjustment for age, body mass index, smoking and leisure-time physical activity did not change these results. The findings were stable over age strata and among present and former baggage handlers.

Conclusions: The risk of musculoskeletal symptoms in six anatomical regions increased with increasing seniority as a baggage handler. This is consistent with the assumption that cumulated heavy lifting may cause chronic or long-lasting musculoskeletal symptoms. However, we cannot exclude that other factors related to baggage handler seniority may explain some of the associations.

\section{INTRODUCTION}

The relation between occupational lifting and musculoskeletal symptoms has been

\section{Strengths and limitations of this study}

- This study includes a large number of baggage handlers with a large variation in seniority.

- We found a high degree of comparability in characteristics of the study and reference group.

- A reference group of working men reduces the risk of healthy worker effect bias in this study.

- Information on exposure and outcome is based on self-reports.

- The interpretation of results might be challenged by general health which is strongly associated with seniority and musculoskeletal symptoms.

examined in several studies with different designs and in different occupational groups. Heavy lifting and lifting in twisted and stooped positions have been found to be the risk factors for developing musculoskeletal disorders in the lower back region, ${ }^{1-9}$ shoulders, ${ }^{10-12}$ hips $^{13}{ }^{14}$ and knees. ${ }^{13}{ }^{15-19}$ However, the degree to which these associations are related to acute reversible effects or to chronic effects from cumulated exposure is not clear, and data on exposure-response associations are sparse. Causal inferences, therefore, remain uncertain. ${ }^{8} 920$

If cumulated heavy lifting in awkward positions causes chronic musculoskeletal symptoms one would expect that seniority in occupations with the same daily exposures over years could serve as a simple proxy measure of cumulated exposure. Baggage handling is characterised by repetitions of the same relatively few work tasks throughout the whole working day. These work tasks are primarily characterised by heavy lifting in awkward positions. ${ }^{21}$ In particular, loading and unloading luggage in compartments of narrow bodied aircrafts are performed in stooped, squatting, sitting or kneeling 
positions in constrained spaces. ${ }^{21} 22$ On an average, a baggage handler at Copenhagen Airport lifts 4-5 tonnes during a normal work shift. The average weight of each lift is around $15 \mathrm{~kg}$ and most of the lifts are performed in awkward positions. The amount of goods lifted by the individual baggage handler has been rather constant over many years (Brauer C, Mikkelsen S, Thygesen LC. Use of company data to assess exposure to manual lifting, personal communication, 2013).

A few epidemiological studies have examined the association between heavy lifting and musculoskeletal symptoms among baggage handlers. ${ }^{22-24}$ Stålhammar et $a l^{22}$ used a questionnaire to measure the occurrence of shoulder, knee and back pain in baggage handlers and found that more than half of the study population reported pain in the shoulders, knees and lower back, even though the population consisted of young men only (mean age 27 years) of whom $59 \%$ had a seniority of less than 5 years. In addition, Undeutsch et $a l^{23} 24$ investigated transport workers in a German airport and found that $66 \%$ reported symptoms in the lower back, $33 \%$ in the neck and $41 \%$ in the arms. Furthermore, they found an association between baggage handler seniority and occurrence of back symptoms. ${ }^{23} 24$ These previous studies were based on limited sample sizes of 78 and 366 baggage handlers, respectively, and no reference group was included in these studies.

The aim of the present study was to examine whether baggage handlers have an increased risk of musculoskeletal symptoms compared with a reference group of men in other unskilled occupations with less heavy work, and whether seniority as a baggage handler is associated with musculoskeletal symptoms.

\section{MATERIAL AND METHODS}

\section{Study population}

Using the electronic employee registers of the two leading handling companies at Copenhagen Airport and the electronic member directory from the local labour union that organises the airport baggage handlers, we identified a group of 4527 persons with occupational codes that indicated employment as a baggage handler anytime between 1983 and 2012. We further used the electronic member directory of unskilled workers in the Greater Copenhagen area, the electronic member directory of the Union of Security Workers and the Copenhagen Airport electronic employee register of security personal in the airport to establish a reference group consisting of 3927 randomly selected men in who within the same period were occupied with other unskilled jobs with less heavy work, for example, cleaning, security and catering.

\section{Data collection}

A questionnaire was delivered to baggage handlers and persons in the reference group who met the following criteria: they were alive in 2012; had permanent residence in Denmark; had an age between 25 and 75 years and had not previously requested not to participate in research projects (an option in Danish civil registration). These criteria were met by 3092 baggage handlers and 2469 in the reference group. The group of baggage handlers consisted of 1140 currently employed and 1952 formerly employed at Copenhagen Airport. The currently employed baggage handlers were asked to fill in the questionnaire at the airport during their working time, while the formerly employed baggage handlers and all individuals in the reference group received the questionnaire by mail. The participants who did not answer the questionnaire within 3 weeks received a phone call and were invited to answer the questionnaire by phone. In total, 2179 baggage handlers (response rate $70.1 \%$ ) and 1710 in the reference group (response rate $68.8 \%$ ) answered the questionnaire.

\section{Measurements of exposure and outcome}

In the questionnaire the participants were asked about their height, weight, date of birth, musculoskeletal symptoms in eight different anatomical regions and lifestyle determinants, such as physical leisure activity and smoking. The questions were all validated questions used in original or slightly modified versions. In addition, baggage handlers were asked supplementary questions about their work as a baggage handler.

In order to validate the information on occupation, participants identified as baggage handlers in the registers and member directory were asked whether they had ever worked as a baggage handler. Only participants who answered in the affirmative were included as baggage handlers in the subsequent analyses, whereas participants who stated that they had never worked as baggage handlers were transferred to the reference group. In total, 352 individuals $(16.2 \%)$ were transferred from the study to the reference group, so that we in the analyses ended up with 1827 baggage handlers and 2062 in the reference group.

Information on baggage handler seniority was measured by the question: For how many years, all together, have you worked as a baggage handler?

Musculoskeletal symptoms were recorded for eight anatomical regions: neck and upper back, lower back, shoulders, elbows, wrists, hips, knees and ankles, and were measured by the question: How much have you been bothered by pain or discomfort in the following body regions during the last 12 months? This was followed by a list of the eight anatomical regions with response categories: not at all, a little/somewhat, quite a lot, and very much. In the analyses the degree of pain was dichotomised into the categories: no complaints which consisted of the categories not at all and a little/somewhat and complaints which consisted of the categories quite a lot and very much.

As potential confounders we included age as a continuous variable. Smoking (never, former smoker, yes), leisure-time physical activity $(<2,2-4,>4 \mathrm{~h} /$ week $)$ and 
body mass index (BMI; $<18.5,18.5-<25,25-<30, \geq 30$ ) were included as categorical variables.

\section{Statistical analyses}

Associations between baggage handler seniority and musculoskeletal symptoms were analysed using three different models. In model 1 we tested differences in regional musculoskeletal symptoms between baggage handlers and the reference group only adjusted for age. In model 2 we further included baggage handler seniority, first as a categorical variable divided into quartiles (the highest quartile covered a large range of seniority and was therefore subdivided into two; model 2.1), and then as a continuous variable with the reference group coded with 0 years of baggage handler seniority (model 2.2). We used the likelihood ratio test to estimate if seniority could be fitted as a linear effect. In all models including seniority as a continuous variable we also included the binary group variable, coded ' 0 ' for the reference group and ' 1 ' for baggage handlers. By this coding, the effect of the seniority variable only refers to baggage handler seniority, and inflation or deflation of effect estimates owing to group differences are avoided. In the final model (model 3) we further included the aforementioned potential confounders . Supplementary analyses were made by adding general health (categories: excellent or very good, good, fair or poor) to the final model (model 4). The data were analysed using logistic regression, SAS V.9.2 (SAS Institute Inc, Cary, North Carolina, USA). Results are presented as OR with 95\% CI.

\section{RESULTS}

Table 1 shows the characteristics of the participants. The age distribution was slightly skewed towards a larger part of older participants in the reference group compared with baggage handlers. The average seniority for baggage handlers was 11 years; $2.4 \%$ had a seniority of less than 1 year and $24.4 \%$ had a seniority of more than 16 years. The Pearson's correlation coefficient between age and baggage handler seniority was 0.56 .

The degree of musculoskeletal symptoms was higher for baggage handlers than for the reference group within all anatomical regions, except for the ankles, and the lower back was the site of most pain in both groups. Furthermore, the height, weight and smoking were similar in the two groups, whereas baggage handlers reported a poorer general health and a higher level of physical leisure activity than the reference group.

Table 2 shows the results of the logistic regression analyses. We found a significantly higher OR of musculoskeletal symptoms in the group of baggage handlers compared with the reference group for all anatomical regions, except for the ankles (model 1). The odds of musculoskeletal symptoms increased systematically with higher categories of baggage handler seniority in six of
Table 1 Participant characteristics and description of variables

\begin{tabular}{|c|c|c|}
\hline & $\begin{array}{l}\text { Baggage } \\
\text { handlers }\end{array}$ & $\begin{array}{l}\text { Reference } \\
\text { group }\end{array}$ \\
\hline $\begin{array}{l}\text { Number of } \\
\text { respondents }\end{array}$ & $1827(47.0)$ & $2059(53.0)$ \\
\hline \multicolumn{3}{|l|}{ Age (years) } \\
\hline $25-34$ & $244(13.6)$ & $227(11.3)$ \\
\hline $35-44$ & $587(32.7)$ & $554(27.5)$ \\
\hline $45-54$ & 644 (35.9) & 679 (33.7) \\
\hline $55-64$ & $236(13.2)$ & 377 (18.7) \\
\hline $65-75$ & $82(4.6)$ & $176(8.8)$ \\
\hline \multicolumn{3}{|l|}{ Seniority (years) } \\
\hline 0 & $0(0.0)$ & $2059(100.0)$ \\
\hline$>0-3$ & $499(28.1)$ & $0(0.0)$ \\
\hline $4-8$ & $404(22.7)$ & $0(0.0)$ \\
\hline $9-16$ & $442(24.9)$ & $0(0.0)$ \\
\hline $17-25$ & $266(15.0)$ & $0(0.0)$ \\
\hline$\geq 26$ & $167(9.4)$ & $0(0.0)$ \\
\hline \multicolumn{3}{|l|}{ Symptoms } \\
\hline Lower back & $553(32.6)$ & $450(23.4)$ \\
\hline Neck/upper back & $353(21.8)$ & $335(17.8)$ \\
\hline Shoulders & $419(25.4)$ & 305 (16.3) \\
\hline Elbows & $174(11.2)$ & $123(6.8)$ \\
\hline Wrists & $185(11.8)$ & $131(7.2)$ \\
\hline Hips & $116(7.6)$ & $111(6.1)$ \\
\hline Knees & 408 (24.3) & $325(17.2)$ \\
\hline Ankles & $127(8.1)$ & $146(8.0)$ \\
\hline Height (cm) (mean) & 181.2 & 180.5 \\
\hline Weight (kg) (mean) & 87.4 & 87.5 \\
\hline \multicolumn{3}{|l|}{ BMI } \\
\hline Underweight & $31(1.7)$ & $66(3.2)$ \\
\hline Normal weight & $644(35.3)$ & 684 (33.2) \\
\hline Overweight & 958 (52.4) & $1046(50.8)$ \\
\hline Obese & $194(10.6)$ & $262(12.7)$ \\
\hline \multicolumn{3}{|l|}{ General health } \\
\hline $\begin{array}{l}\text { Excellent/very } \\
\text { good }\end{array}$ & $692(38.3)$ & $897(44.0)$ \\
\hline Good & $725(40.1)$ & 834 (40.9) \\
\hline Fair/poor & 394 (21.6) & 307 (15.1) \\
\hline \multicolumn{3}{|l|}{ Smoking } \\
\hline No & $721(39.8)$ & 704 (34.4) \\
\hline Former & $590(32.6)$ & 724 (35.3) \\
\hline Yes & $501(27.6)$ & $621(30.3)$ \\
\hline \multicolumn{3}{|c|}{ Physical leisure activity (h/week) } \\
\hline$<2$ & $179(9.9)$ & $721(39.8)$ \\
\hline $2-4$ & $618(34.2)$ & $590(32.6)$ \\
\hline$>4$ & $1008(55.8)$ & $501(27.7)$ \\
\hline
\end{tabular}

Values are numbers (percentages) unless stated otherwise. $\mathrm{BMI}$, body mass index.

the anatomical regions: the lower back, neck and upper back, shoulders, elbows, wrists and knees (model 2.1).

The likelihood ratio test showed that the effect of baggage handler seniority could be fitted as a linear effect for all regions except for the hips, and hence baggage handler seniority was included continuously in the final model (model 3). When baggage handler seniority was included continuously in the model (model 2.2), the 
Table 2 OR $(95 \% \mathrm{Cl})$ for musculoskeletal symptoms within the last 12 months according to occupation and baggage handler seniority for baggage handlers and a reference group with less heavy work

\section{OR (Cl 95\%)}

Lower back

Neck/upper back Shoulders

Elbows

Wrists

Hips

Knees

Model 1

No

Yes
Model 2.1

Seniority (years)

0 (reference)

$>0-3$

$4-8$

9-16

$17-25$

$>26$

Model 2.2

Baggage handler

No

Yeniority (per 10 years)

Seniority (per
Reference

Baggage handler

Model 3

Baggage handler

No

Seniority (per 10 years)

Reference

Baggage handle

Age (per 10 years)

Smoking

No

Former smoke

Yes

Physical activity (h/week)

$<2$
$2-4$

$2-4$

$>4$

Underweight

Normal weight

Obese

Overweight

Model 4

Baggage handler

No

Yeniority (per 10 years)

Reference

Baggage handlers

$1.64(1.42$ to 1.91$) \quad 1.34(1.13$ to 1.59$) \quad 1.8$.

$\begin{array}{lll}\mathrm{N} & & \\ 2059 & 1 & 1 \\ 499 & 1.09(0.85 \text { to } 1.38) & 0.97\end{array}$

$499 \quad 1.09(0.85$ to 1.38$) \quad 0.97(0.73$ to 1.28$)(1.21)$

$404 \quad 1.25(0.96$ to 1.61$) \quad 1.15(0.85$ to 1.53$)$

1.91 (1.52 to 2.39 ) $\quad 1.45(1.11$ to 1.88$)$

$2.41(1.82$ to 3.18$) \quad 1.70(1.24$ to 2.32$)$

3.02 (2.12 to 4.30$) \quad 2.26$ (1.53 to 3.30$)$

$1.10(0.89$ to 1.35$)$

$1.42(1.26$ to 1.61$) \quad 1.32(1.16$ to 1.52$) \quad 1.27(1.12$ to 1.45$) \quad 1.55(1.29$ to 1.85$)$

1

$\begin{array}{lllll}1.38(1.22 \text { to } 1.56) & 1.30(1.14 \text { to } 1.50) & 1.27(1.11 \text { to } 1.45) & 1.53(1.28 \text { to } 1.83) & 1.33(1.11 \text { to } 1.60) \\ 1.06(0.98 \text { to } 1.15) & 1.01(0.92 \text { to } 1.11) & 1.04(0.95 \text { to } 1.14) & 1.07(0.93 \text { to } 1.23) & 1.07(0.94 \text { to } 1.21)\end{array}$

1

$1.02(0.85$ to 1.23$)$

1.28 (1.05 to 1.55$)$

$1.16(0.94$ to 1.43$)$

1.20 (0.96 to 1.49$)$

$\stackrel{1}{0.70}(0.54$ to 0.91$)$

0.94 ( 0.74 to 1.21$)$

$0.75(0.59$ to 0.96$)$

$0.56(0.43$ to 0.72$)$

0.94 (0.44 to 1.85 )

0.81 ( 0.41 to 1.50$)$

1.11 (0.94 to 1.32$)$ $1.64(1.28$ to 2.10$)$

$1.46(1.10$ to 1.95$)$

$1.21(0.92$ to 1.60$)$ 1.57 (1.18 to 2.06$)$ 2.27 (1.77 to 2.91)

$2.31(1.57$ to 3.37$)$

$0.82(0.50$ to 1.28$)$ 1.50 (0.98 to 2.23$)$ $2.19(1.53$ to 3.09$)$ 2.93 (1.74 to 4.76$)$

$1.26(0.84$ to 1.85$)$ 1.46 (0.96 to 2.16$)$ 1.94 (1.35 to 2.74$)$ 2.33 ( 1.54 to 3.46$)$

$3.15(1.93$ to 5.01$)$

1.22 (0.87 to 1.70$)$

$1.38(1.16$ to 1.64$)$

$1.03(0.68$ to 1.55$)$

1

$1.27(1.03$ to 1.56$) \quad 1.30(1.14$ to 1.48$)$

$1.30(1.14$ to 1.48$) \quad 1.30(1.07$ to 1.57$)$

1

1.35 (1.06 to 1.71$) \quad 0.92(0.62$ to 1.34$)$

$1.26(1.01$ to 1.44$) \quad 1.21$ (0.99 to 1.49$)$ $1.17(1.07$ to 1.28$) \quad 1.36(1.19$ to 1.56$)$

1

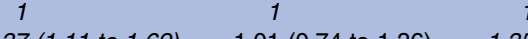

$1.37(1.11$ to 1.69$) \quad 1.01(0.74$ to 1.36$) \quad 1.35(1.01$ to 1.82$)$

$1.29(0.95$ to 1.76$) \quad 1.30(0.96$ to 1.77$)$

1

$89(0.68$ to 1.18$) \quad 1.00(0.67$ to 1.50$) \quad 0.96(0.66$ to 1.40$)$

$0.94(0.72$ to 1.24$)-0.93(0.63$ to 1.39$)$

0.74 (0.51 to 1.09$)$

$\begin{array}{lll}0.74 \text { (0.33 to } 1.68) & 0.52(0.12 \text { to } 1.49) & 1.73(0.76 \text { to } 3.56) \\ 1 & 1 & 1\end{array}$

1.36 (1.12 to 1.65$) \quad 0.86(0.65$ to 1.33$) \quad 0.79(0.61$ to 1.04$)$

1.28 (0.86 to 1.88$) \quad 1.40$ (0.96 to 2.01$)$

Model 1: Baggage handler (yes/no) and age.

Model 2.1: Baggage handler (yes/no), baggage handler seniority (categorical) and age.

Model 2.2: Baggage handler (yes/no), baggage handler seniority (continuous) and age

Model 3: Baggage handler (yes/no), baggage handler seniority (continuous), age, height, weight, smoking, physical leisure activity

Model 4: Baggage handler (yes/no), baggage handler seniority (continuous), age, height, weight, smoking, physical leisure activity, general health

BMI, body mass index.

Italic typeface indicates significance at $p<0.05$. 
effect of baggage handler (yes/no) diminished and became insignificant for all regions, except for the shoulders (OR 1.37, 95\% CI 1.08 to 1.72) while the linear effect of baggage handler seniority was statistically significant in all of the anatomical regions. Thus, the higher prevalence of musculoskeletal symptoms among baggage handlers was, to a large extent, explained by seniority as a baggage handler.

Model 3 shows that when age, BMI, smoking and physical leisure activity were added in the model, the effect estimates decreased but remained substantial and significant for all of the regions, except for the ankles. For example, for every 10 year of baggage handler seniority the odds of symptoms in the lower back increased by $38 \%$ (OR 1.38, $95 \%$ CI 1.22 to 1.56 ), the odds of symptoms in the elbows increased by $53 \%$ (OR 1.53, 95\% CI 1.28 to 1.83 ) and the odds of symptoms in the wrists increased by 33\% (OR $1.33,95 \%$ CI 1.11 to 1.60$)$. Furthermore, model 3 shows that only symptoms in the knees and ankles were significantly affected by age after adjustment for seniority. In general, the covariates had the same effect on musculoskeletal symptoms as known from former studies: physical leisure activity decreased the odds of pain whereas smoking $^{45}$ and a high BMI ${ }^{13161719}$ increased the odds of pain.

Additional analyses for the hips showed that the risks of symptoms in the first four categories of seniority (in model 2.1) were not significantly different and could be combined into one category (0-16 years) without changing the fit of the model significantly (data not shown). This indicates that the risk of hip symptoms did not significantly increase until at least 26 years of baggage handler seniority.

In all of the adjusted analyses we tested adjustment for height and weight instead of BMI changed the estimates. Also, we tested for interactions between height and weight. None of these variations changed the estimates substantially. Furthermore, stratified analyses on current versus former baggage handlers showed that the effects of seniority reported in model 3 remained significant for both groups within all anatomical regions except for the knees (figure 1).

\section{Supplementary analyses}

As noted in table 1, self-reported general health of baggage handlers was poorer than that of the reference group and further analysis revealed that this relation increased with baggage handler seniority. Furthermore, general health was associated with musculoskeletal symptoms. We tried to examine whether the relation between general health and baggage handler seniority disappeared if we adjusted for number of regions with symptoms. In this analysis, we further included age and the other covariates in the final model on regional pain and seniority (data not shown). By doing so the relation between general health and seniority still persisted, indicating that seniority and thereby cumulated work factors are also related to other health effects than musculoskeletal symptoms and that the linear relation between baggage handler seniority and symptoms might-to some extent-be explained by general health. In order to assess the maximal potential bias, which could be related to general health, we added general health as a covariate in the final model (model 4). The linear relation between seniority and pain persisted for all regions and was still significant for lower back (OR 1.20, 95\% CI 1.05 to 1.38 ) and elbows (OR 1.43, 95\% CI 1.18 to 1.72), and the lower confidence limit for the other regions was only slightly below unity in most regions.

\section{DISCUSSION}

We found that the ORs of self-reported musculoskeletal symptoms in the neck and upper back, lower back, shoulders, elbows, wrists, hips and knees were significantly higher in baggage handlers than in a reference

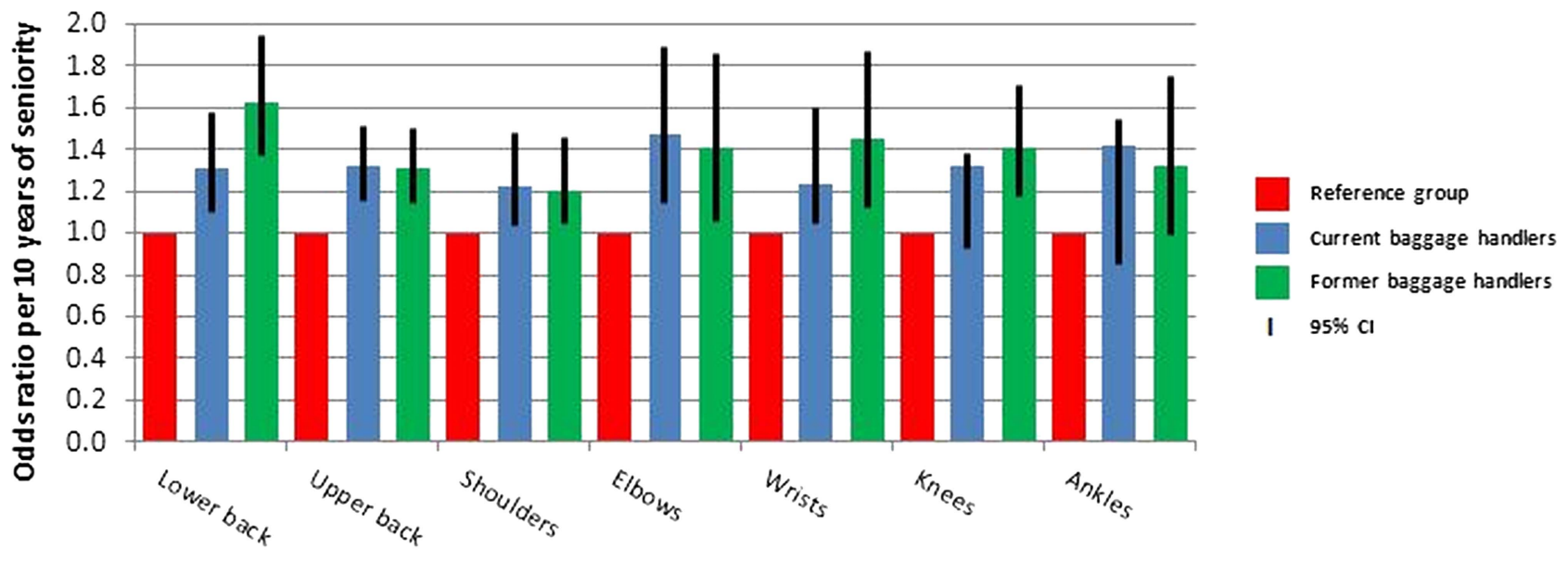
Adjusted for baggage handler (Yes/no), age, height, weight, smoking and
physical leisure activity

Figure 1 OR of muscucoskeletal symptoms for current and former baggage handlers compared with the reference group. 
group of men in other unskilled occupations with less heavy work. These differences between the groups were, to a great extent, attributable to length of employment as a baggage handler, indicating that the study and reference groups were basically comparable in relation to reporting of musculoskeletal symptoms.

Baggage handler seniority was significantly, positively associated with musculoskeletal symptoms in all of the measured anatomical regions, except for the ankles, and a significant, linear relationship was found for the neck and upper back, lower back, shoulders, elbows, wrists and knees. However, the pattern for the hips was irregular and only significantly increased compared with the control group for baggage handlers with more than 26 years of seniority. These results may suggest that cumulated heavy lifting in awkward positions does not only affect the lower back, knees and shoulders, as indicated by previous studies but also other anatomical regions, such as the wrists and elbows. As we do not expect cumulated exposure to be associated with acute reversible musculoskeletal symptoms, the implication is that long-lasting daily exposures to heavy lifting in awkward positions may cause chronic or long-lasting adverse effects on musculoskeletal health in several body regions. This interpretation is supported by similar relations between seniority and musculoskeletal symptoms among presently and previously employed baggage handlers.

Our results are in line with the study of Undeutsch et $a l^{23}$ that found an age-adjusted association between seniority as a baggage handler and occurrence of back symptoms. However, the present study is the first to show a linear relationship between baggage handler seniority and self-reported pain in a number of other anatomical regions.

In this study, we found a linear association between baggage handler seniority and musculoskeletal symptoms in six of eight anatomical regions, although some of the regions are not normally assumed to be affected by heavy lifting, for example, the wrists. This lack of regional specificity in the relation between baggage handler seniority and pain may be seen as a weakness in the causal interpretation of our findings. However, heavy lifting in awkward positions implies biomechanical loads on all body parts, and short-term exposure to baggage handling is associated with acute pain in most of the anatomical regions included in our study. ${ }^{25-28}$ Thus, if repeated acute pain plays a role in the development of chronic pain, the lack of specificity of the relationship between regional pain and seniority may not be an important issue in the interpretation of our results. Furthermore, it is well known that the reporting of pain in one anatomical region is associated with increased pain reporting from adjacent and contralateral regions, possibly due to pain processing in the central nervous system. $^{29} 30$

One may also wonder about the linear effect of seniority from even low levels, as it might be expected that the effects of cumulated exposure would occur only after a longer period of exposure. The higher OR of pain in baggage handlers with increasing seniority could possibly be explained by the combination of recurrent episodes of acute pain from soft tissue strains and more chronic pain from degenerative changes in the joints and tendons at higher seniority. The contribution by different mechanisms might also differ between regions and could possibly explain the somewhat different findings for the hip region. However, the mechanisms leading to long-lasting or chronic musculoskeletal pain are unknown.

\section{Limitations}

It may be a limitation that information on seniority as well as musculoskeletal symptoms was based on selfreports, which may involve recall bias and differential misclassification. However, we consider seniority to be a factual information with an expected high level of accuracy. If our findings of a linear relationship between seniority and musculoskeletal symptoms were attributable to misclassification of pain or seniority, baggage handlers should consistently and increasingly overestimate either their pain by increasing seniority or their seniority by increasing levels of pain. We cannot exclude such biases but consider them as unlikely explanations of our results.

Another limitation that may challenge the interpretation of our results is that general health was strongly associated with seniority and pain. Our supplementary analyses showed that the linear relation between baggage handler seniority and pain could, to some extent, be explained by general health (table 2, model 4). However, it is important to consider the possible pathways between baggage handler seniority, general health and regional pain. One pathway is that baggage handler seniority reflects cumulated exposure to heavy lifting, causing regional musculoskeletal pain which leads to a feeling of poorer general health. In this case, the relation between seniority and musculoskeletal pain should not be adjusted for effects of general health. However, an alternative pathway might also exist: the poorer general health that follows with baggage handler seniority could result from other health affecting factors than heavy lifting that cumulates with length of employment, for example, particulate air pollution or psychosocial work conditions. This could be associated with more unspecific symptom reporting in general, including reporting of diffuse regional pain. If this is the case, the associations between baggage handler seniority and pain could be explained by a poorer general health caused by factors additional to heavy lifting. However, even if we assume the last mentioned pathway to be the dominating-and thereby our supplementary analyses to reflect the true associations-the pattern of associations between seniority and pain persisted for all regions and was still significant for lower back and elbows, while the lower 
confidence limit for the other regions was only slightly below unity.

Furthermore, we measured only the associations between baggage handler seniority and current musculoskeletal symptoms without considering time for onset of the symptoms or the way the symptoms started. We assume that the symptoms are caused by cumulative hard musculoskeletal demands but it could be caused by accidents at work or in leisure time or even have occurred before the employment as a baggage handler. Finally, in our analyses, we assume that exposure to heavy lifting has been constant over the years, without considering the changes in external factors that might have affected the risk of musculoskeletal disorders, such as air traffic, work schedules and the introduction of assistive equipment to reduce the manual workload. However, data on flights, goods and baggage handlers from Copenhagen Airport show that even when considering these factors, the average of goods lifted by the individual baggage handler seems rather constant during the study period (Brauer et al, unpublished).

The strengths of this study are the large number of currently and formerly employed baggage handlers with a large variation in seniority. Furthermore, all the covariates in the analyses had the expected influence on pain which corroborates the validity in data. In addition, our data showed a high degree of comparability in characteristics of the study and reference group, and the inclusion of a reference group consisting of working men only reduces the influence of healthy worker effect bias. ${ }^{31-33}$ However, the observed associations could not be explained by healthy worker selection; if musculoskeletal symptoms led some baggage handlers to leave their jobs, the exposureresponse relationship with seniority would only be weakened. Similarly, if some of the reference groups had also at sometimes held heavy manual jobs, the effect would have been to reduce the strength of the associations.

\section{CONCLUSION}

We conclude that baggage handlers had a significantly higher risk of musculoskeletal symptoms than a reference group with less heavy work. This difference was, to a large extent, explained by seniority as a baggage handler. Furthermore, we found a strong linear association between regional musculoskeletal symptoms and seniority which is consistent with a long-lasting or chronic effect of cumulated exposure to heavy lifting. However, we cannot exclude that other factors related to baggage handler seniority may explain some of the associations. To pursue this issue further, future research should include information on onset and cause of pain, and estimates of individual differences in the amount and frequency of heavy lifting.

Contributors SHB drafted the article and all authors revised it critically for important intellectual content, and all authors approved the final version to be published. SHB, HK, CB, LCT, EBS, TA, JPB and SM contributed in study conception and design. SHB, KLM and HK contributed in acquisition of the data. SHB, SM, CB, LCT and JPB contributed in interpretation of data. SHB and $\mathrm{SM}$ contributed in statistical analyses.

Funding This research was financially supported by the Danish Working Environment Fund (grant number 24-2011-03) and the Danish Rheumatism Association (grant number R101-A2003).

\section{Competing interests None.}

Ethical approval The study was notified to the Scientific Ethical Committee, County of Copenhagen (journal no: H-4-2011-125) but returned without review because Danish law does not require that questionnaire studies are approved be an ethical committee.

Patient consent Obtained.

Provenance and peer review Not commissioned; externally peer reviewed.

Data sharing statement No additional data are available.

Open Access This is an Open Access article distributed in accordance with the Creative Commons Attribution Non Commercial (CC BY-NC 3.0) license, which permits others to distribute, remix, adapt, build upon this work noncommercially, and license their derivative works on different terms, provided the original work is properly cited and the use is non-commercial. See: http:// creativecommons.org/licenses/by-nc/3.0/

\section{REFERENCES}

1. Seidler A, Bolm-Audorff U, Siol T, et al. Occupational risk factors for symptomatic lumbar disc herniation; a case-control study. Occup Environ Med 2003;60:821-30.

2. Seidler A, Bergmann A, Jager $M$, et al. Cumulative occupational lumbar load and lumbar disc disease-results of a German multi-center case-control study (EPILIFT). BMC Musculoskelet Disord 2009;10:48.

3. Leino-Arjas P, Kaila-Kangas L, Kauppinen T, et al. Occupational exposures and inpatient hospital care for lumbar intervertebral disc disorders among Finns. Am J Ind Med 2004;46:513-20.

4. Burdorf A, Sorock G. Positive and negative evidence of risk factors for back disorders. Scand J Work Environ Health 1997;23:243-56.

5. Gerr F, Mani L. Work-related low back pain. Prim Care 2000;27:865-76

6. Seidler A, Bolm-Audorff U, Heiskel H, et al. The role of cumulative physical work load in lumbar spine disease: risk factors for lumbar osteochondrosis and spondylosis associated with chronic complaints. Occup Environ Med 2001;58:735-46.

7. Moshe S, Levin M. [Occupational aspects of low back pain]. Harefuah 2005;144:492-6, 526.

8. Videman T, Battie MC. The influence of occupation on lumbar degeneration. Spine (Phila Pa 1976) 1999;24:1164-8.

9. Wai EK, Roffey DM, Bishop P, et al. Causal assessment of occupational lifting and low back pain: results of a systematic review. Spine J 2010;10:554-66.

10. Andersen $\mathrm{JH}$, Kaergaard A, Mikkelsen S, et al. Risk factors in the onset of neck/shoulder pain in a prospective study of workers in industrial and service companies. Occup Environ Med 2003;60:649-54.

11. van der Windt DA, Thomas E, Pope DP, et al. Occupational risk factors for shoulder pain: a systematic review. Occup Environ Med 2000;57:433-42.

12. Miranda H, Punnett L, Viikari-Juntura E, et al. Physical work and chronic shoulder disorder. Results of a prospective population-based study. Ann Rheum Dis 2008;67:218-23.

13. Fransen $M$, Agaliotis $M$, Bridgett $L$, et al. Hip and knee pain: role of occupational factors. Best Pract Res Clin Rheumatol 2011;25:81-101.

14. Jensen LK. Hip osteoarthritis: influence of work with heavy lifting, climbing stairs or ladders, or combining kneeling/squatting with heavy lifting. Occup Environ Med 2008;65:6-19.

15. Amin S, Goggins J, Niu J, et al. Occupation-related squatting, kneeling, and heavy lifting and the knee joint: a magnetic resonance imaging-based study in men. J Rheumatol 2008;35:1645-9.

16. Vrezas I, Elsner G, Bolm-Audorff U, et al. Case-control study of knee osteoarthritis and lifestyle factors considering their interaction with physical workload. Int Arch Occup Environ Health 2010;83:291-300.

17. Palmer KT. Occupational activities and osteoarthritis of the knee. $\mathrm{Br}$ Med Bull 2012;102:147-70.

18. Reid CR, Bush PM, Cummings $\mathrm{NH}$, et al. A review of occupational knee disorders. J Occup Rehabil 2010;20:489-501. 
19. Jensen LK. Knee osteoarthritis: influence of work involving heavy lifting, kneeling, climbing stairs or ladders, or kneeling/squatting combined with heavy lifting. Occup Environ Med 2008;65:72-89.

20. da Costa BR, Vieira ER. Risk factors for work-related musculoskeletal disorders: a systematic review of recent longitudinal studies. Am J Ind Med 2010;53:285-323.

21. Tapley S, Riley D. Baggage handling in narrow-bodied aircraft: identification and assessment of musculoskeletal injury risk factors. In: East and South East Specialist Group, ed. Health and Safty Executive, 2005. http://www.hse.gov.uk/airtransport/docs/baggage.pdf

22. Stålhammar HR, Leskinen TP, Kuorinka IA, et al. Postural, epidemiological and biomechanical analysis of luggage handling in an aircraft luggage compartment. Appl Ergon 1986;17:177-83.

23. Undeutsch K, Gartner KH, Luopajarvi T, et al. Back complaints and findings in transport workers performing physically heavy work. Scand J Work Environ Health 1982;8(Suppl 1):92-6.

24. Undeutsch K, Kupper R, Lowenthal I, et al. [Occupational health studies on airport transport workers. III. Musculoskeletal complaints and orthopedic disorders of airport transport workers (author's transl)]. Int Arch Occup Environ Health 1982;50:59-75.

25. Harkness EF, Macfarlane GJ, Nahit ES, et al. Mechanical and psychosocial factors predict new onset shoulder pain: a prospective cohort study of newly employed workers. Occup Environ Med 2003;60:850-7.
26. Harkness EF, Macfarlane GJ, Nahit ES, et al. Risk factors for new-onset low back pain amongst cohorts of newly employed workers. Rheumatology (Oxford) 2003;42:959-68.

27. Jones GT, Harkness EF, Nahit ES, et al. Predicting the onset of knee pain: results from a 2-year prospective study of new workers. Ann Rheum Dis 2007:66:400-6.

28. Nahit ES, Taylor S, Hunt IM, et al. Predicting the onset of forearm pain: a prospective study across 12 occupational groups. Arthritis Rheum 2003;49:519-25.

29. Andersen LL, Andersen $\mathrm{CH}$, Sundstrup E, et al. Central adaptation of pain perception in response to rehabilitation of musculoskeletal pain: randomized controlled trial. Pain Physician 2012;15:385-94.

30. O'Neill S, Manniche C, Graven-Nielsen T, et al. Generalized deep-tissue hyperalgesia in patients with chronic low-back pain. Eur J Pain 2007;11:415-20.

31. Checkoway H, Pearce N, Dement JM. Design and conduct of occupational epidemiology studies: I. Design aspects of cohort studies. Am J Ind Med 1989;15:363-73.

32. Thygesen LC, Hvidtfeldt UA, Mikkelsen S, et al. Quantification of the healthy worker effect: a nationwide cohort study among electricians in Denmark. BMC Public Health 2011;11:571.

33. Steenland K, Stayner L. The importance of employment status in occupational cohort mortality studies. Epidemiology 1991; 2:418-23. 